\title{
Anti-inflammatory effects of betaine on AOM/DSS-induced colon tumorigenesis in ICR male mice
}

\author{
DONG HWAN KIM ${ }^{1}$, BOKYUNG SUNG ${ }^{1}$, YONG JUNG KANG ${ }^{1}$, JUNG YOON JANG ${ }^{1}$, \\ SEONG YEON HWANG ${ }^{1}$, YUJIN LEE ${ }^{1}$, MINJUNG KIM ${ }^{1}$, EUNOK IM ${ }^{1}$, JEONG-HYUN YOON $^{1}$, \\ CHEOL MIN KIM ${ }^{2,3}$, HAE YOUNG CHUNG ${ }^{1}$ and NAM DEUK KIM ${ }^{1,2}$ \\ ${ }^{1}$ Department of Pharmacy, Molecular Inflammation Research Center for Aging Intervention, \\ ${ }^{2}$ Research Center for Anti-Aging Technology Development, Pusan National University, Busan 609-735; \\ ${ }^{3}$ Department of Biochemistry, Pusan National University School of Medicine, Yangsan 626-770, Republic of Korea
}

Received April 14, 2014; Accepted May 30, 2014

DOI: $10.3892 /$ ijo.2014.2515

\begin{abstract}
Betaine is an important human nutrient obtained from various foods and studies in animals and humans have provided results suggesting their pathogenesis of various chronic diseases and points to a role in risk assessment and disease prevention. However, the molecular mechanisms of its activity remain poorly understood and warrant further investigation. This study was performed to investigate the anti-inflammation and tumor preventing capacity of betaine on colitis-associated cancer in mice. In in vivo experiments, we induced colon tumors in mice by azoxymethane (AOM) and dextran sulfate sodium (DSS) and evaluated the effects of betaine on tumor growth. Administration with betaine significantly decreased the incidence of tumor formation with downregulation of inflammation. Treatment with betaine inhibited ROS generation and GSSG concentration in colonic mucosa. Based on the qPCR data, administration of betaine inhibited inflammatory cytokines such TNF- $\alpha$, IL-6, iNOS and COX-2. In in vitro experiments, LPS-induced NF- $\mathrm{kB}$ and inflammatory-related cytokines were inhibited by betaine treatment in RAW 264.7 murine macrophage cells. Our findings suggest that betaine is one of the candidates for the prevention of inflammation-associated colon carcinogenesis.
\end{abstract}

\section{Introduction}

Cancer of colon and rectum (colorectal cancer, CRC) is a malignant neoplasm arising from the lining of the large intestine. Patients with inflammatory bowel disease (IBD), including

Correspondence to: Dr Nam Deuk Kim, Department of Pharmacy, College of Pharmacy, Pusan National University, Busan 609-735, Republic of Korea

E-mail: nadkim@pusan.ac.kr

Key words: betaine, inflammatory bowel disease, colon cancer, azoxymethane/dextran sulfate sodium model both ulcerative colitis (UC) and Crohn's disease (CD), are at increased risk of developing CRC (1). CRC is a worldwide health-care problem with a continually increasing incidence. In Asia, IBD was considered rare until two decades ago (2), but recent population-based and referral center cohorts have shown a rising incidence and prevalence of IBD in Asia (3). Importantly, the incidence and prevalence of $\mathrm{CD}$ and $\mathrm{UC}$ in Korea are still lower than those in Western countries, but are rapidly increasing (4). IBD can occur in combination of defected immune response, luminal, environment and genetic factors including tumor necrosis factor (TNF)- $\alpha$. The evaluated level of TNF- $\alpha$ was especially found in the blood, intestinal mucosa and stools of patients with IBD. In addition to TNF- $\alpha$, the increases of other pro-inflammatory mediators have been observed in stools and rectal dialysates from patients with IBD as well (5). The existing data revealed that TNF- $\alpha$ inhibitors are increasingly being used in IBD-related studies and patients. The most commonly used biologics in IBD are TNF- $\alpha$ antibodies, such as infliximab, a chimeric IgG1 monoclonal antibody; adalimumab, a human monoclonal IgG1 antibody; and certolizumab pegol, a pegylated Fab fragment of a humanized IgG4 isotype monoclonal antibody (6). Even though these TNF inhibitors may increase the risk of tuberculosis, varicella and other opportunistic infections, there is little evidence suggesting that anti-TNF agents specifically raise the overall risk of serious infections. Similarly, there is little evidence that TNF antagonists raise the risk of developing malignancy over and above the risks from concomitant therapies and the underlying disease process (7). Therefore, more studies are needed on the use of TNF inhibitors in patients with IBD.

Betaine is an essential biochemical molecule of the methionine/homeocysteine cycle and is synthesized by conversion of choline. It was first discovered in the juice of sugar beets (Beta vulgaris) in the 19th century (8), and since then has been found in various microorganisms, plants and animals $(9,10)$. It plays central roles in choline-mediated one-carbon metabolism, structural integrity and signaling functions of cell membranes, and neurotransmitter synthesis (11). Previous studies showed dietary choline and betaine intakes and associations with inflammatory markers in healthy free-eating adults enrolled 
in the ATTICA study. Moreover, betaine and choline may be involved in reducing inflammation, including their important role as a source of one-carbon units for the metabolism of homocysteine. In highest tertile for dietary intake of choline and betaine had significantly lower plasma C-reactive protein, interleukin (IL)-6, and TNF- $\alpha$ concentration than did persons in the lowest tertile of intake (12).

Previous studies have shown that betaine has anti-inflammatory activity through inhibition of reactive species (RS) and modulation of reduced glutathione (GSH)/oxidized glutathione (GSSG) ratio in aging process both in vitro and in vivo studies (13-15). However, the protective role of betaine on the expression and regulation of inflammatory mediators associated with colon cancer has not explored yet. Hence, in the present study we aimed to evaluate the anti-inflammatory effects of betaine on AOM/DSS-induced colitis-associated colon cancer in mice. We demonstrate that betaine is a potent anti-inflammatory agent that may act through the inactivation of inflammatory cytokines.

\section{Materials and methods}

Chemicals. Betaine was purchased from Sigma-Aldrich Co. (St. Louis, MO, USA). Betaine was freshly prepared before each experiment and was solubilized with phosphate buffered saline (PBS).

Animal study. The animal protocol used in this study has been reviewed by the Pusan National University-Institutional Animal Care and Use Committee (PNU-IACUC, Busan, Korea) on their ethical procedures and scientific care, and it has been approved (PNU-2013-0318) as previously described (16). Five-week-old male ICR mice were purchased from Samtako Co., Ltd. (Osan, Korea). All animals were housed in plastic cages (4 mice/cage) and had free access to drinking water and a basal diet (Formula M07; Feed Lab, Guri, Korea) ad libitum, under controlled conditions of humidity $(50 \pm 10 \%)$, light $(12 / 12 \mathrm{~h}$ light/dark cycle), and temperature $\left(23 \pm 1^{\circ} \mathrm{C}\right)$. After arrival, the animals were quarantined for 7 days, and then randomized by body weights into experimental and control groups. A colonic carcinogen AOM was purchased from Sigma-Aldrich. DSS with a molecular weight of 36,000-50,000 (cat. no. 160110) was purchased from MP Biomedicals, LLC (Aurora, $\mathrm{OH}$, USA). The experimental protocol is shown in Fig. 1A. Animals were divided into four experimental groups and control group ( $n=15$ per group). Group 1 was the control. Animals in group 2 through 5 were given a single intraperitoneal injection of AOM (10 mg/kg body weight). Seven days after the AOM injection, animals received $2 \%$ DSS $(\mathrm{w} / \mathrm{v})$ in the drinking water for 7 days. Seven days after the DSS treatment, betaine-containing diets were started. Subsequently, groups 3 through 5 received the diets containing 1,5 and $10 \mathrm{mg} / \mathrm{kg}$ betaine for 16 weeks, respectively. All animals were sacrificed at week 16 after administration of betaine. At sacrifice, a complete necropsy was performed on all mice. Histopathological examination was performed on paraffin-embedded sections after hematoxylin and eosin (H\&E) staining.

GSH assay. To analyze the total glutathione level, using OxiSelect $^{\mathrm{TM}}$ Total Glutathione (GSSG/GSH) Assay kit (Cell
Biolabs, Inc., San Diego, CA, USA) according to company protocol. GSH reductase solution $(25 \mu \mathrm{l})$, was added to each well in a 96-well plate and $25 \mu 1$ of the NADPH was added onto it; $100 \mu \mathrm{l}$ of the prepared GSH standards or samples was added to each well and mixed thoroughly. Then $50 \mu \mathrm{l}$ of the chromogen was added and the absorbance was read at $405 \mathrm{~nm}$ by using a multi-well reader (Thermo Fisher Scientific, Vantaa, Finland).

Assessment of reactive species $(R S)$ generation. RS generation was measured as previously described using a fluorescence probe (17). Briefly, 2',7'-dichlorofluorescin diacetate (DCF-DA; final concentration $2.5 \mu \mathrm{M}$ ) was added to homogenates and the changes in fluorescence intensity were measured every $5 \mathrm{~min}$ for 30 min using a fluorescence plate reader (GENios, Tecan Instruments, Salzburg, Austria) at excitation and emission wavelengths of 485 and $530 \mathrm{~nm}$, respectively.

Total RNA extraction and quantitative real-time PCR. Total RNA was extracted from colonic mucosa using the TRIzol (Qiagen, Tokyo, Japan) according to the manufacturer's protocol. The cDNA was then synthesized from total RNA using TOPscript ${ }^{\mathrm{TM}}$ RT DryMix (Enzynomics, Daejeon, Korea). A quantitative real-time PCR (qPCR) analysis of individual cDNA was performed with Takara TP800 instrument (Takara Bio Inc., Shiga, Japan) using SYBR-Green gene expression assay (Enzynomics). The primers used for each reverse transcription-polymerase chain reaction reactions are as follows: GAPDH, 5'-aactttggcattgtggaagg-3' and 5'-acacattgggggtaggaaca-3'; TNF- $\alpha, 5^{\prime}$-'cgtcagccgatttgctatct-3' and 5'-cggactccgcaaagtctaag-3'; inducible nitric oxide synthase (iNOS), 5'-ctcactgggacagcacagaa-3' and 5'-gcttgtctctgggtcct ctg-3'; cyclooxygenase (COX)-2; 5'-gctgtacaagcagtggcaaa-3' and 5'-cccaaagatagcatctgga-3'; IL-6, 5'-agttgcettcttgggactga-3' and 5'-ttctgcaagtgcatcatcgt-3' (forward and reverse, respectively). The PCR cycling conditions were $95^{\circ} \mathrm{C}$ for $15 \mathrm{~min}$, followed by 40 cycles of $95^{\circ} \mathrm{C}$ for $15 \mathrm{sec}$ and $54^{\circ} \mathrm{C}$ for $10 \mathrm{sec}$ and $72^{\circ} \mathrm{C}$ for $20 \mathrm{sec}$. The expression level of each gene was normalized to the GAPDH expression level. Each assay was performed in triplicate and the average was calculated.

Cell culture and cell viability assay. The murine macrophage RAW 264.7 cells were obtained from American Type Culture Collection (Manassas, VA, USA) and cultured in DMEM (HyClone, Logan, UT, USA) supplemented with $10 \%$ fetal bovine serum (FBS, HyClone), $100 \mathrm{U} / \mathrm{ml}$ penicillin and $100 \mu \mathrm{g} / \mathrm{ml}$ streptomycin (HyClone) at $37^{\circ} \mathrm{C}$ in a humidified $5 \% \mathrm{CO}_{2}$. Cell viability was determined by MTT assay. For the MTT assay, RAW 264.7 cells were seeded in a 24-well culture plate at a density of $4 \times 10^{4}$ cells/well, cultured for $24 \mathrm{~h}$ in the growth media and then treated with or without betaine for the indicated concentrations. The cells were incubated with $0.5 \mathrm{mg} / \mathrm{ml}$ MTT [3-(4,5-dimethylthiazol-2-yl)-2,5-diphenyltetrazolium bromide] (Sigma-Aldrich) at $37^{\circ} \mathrm{C}$ for $2 \mathrm{~h}$. The formazan granules generated by the live cells were dissolved in DMSO and the absorbance at $540 \mathrm{~nm}$ was monitored by using a multi-well reader (Thermo Fisher Scientific).

Western blot analysis. The cells were treated under the appropriate conditions, harvested and washed with cold PBS and 
were lysed in lysis buffer [ $40 \mathrm{mM}$ Tris ( $\mathrm{pH} 8.0), 120 \mathrm{mM} \mathrm{NaCl}$, $0.5 \% \mathrm{NP}-40,0.1 \mathrm{mM}$ sodium orthovanadate, $2 \mu \mathrm{g} / \mathrm{ml}$ aprotinin, $2 \mu \mathrm{g} / \mathrm{ml}$ leupeptin and $100 \mu \mathrm{g} / \mathrm{ml}$ phenymethylsulfonyl fluoride (PMSF)]. The supernatant was collected and protein concentrations were measured (Bio-Rad, Hercules, CA, USA). Protein extracts were denatured by boiling at $100^{\circ} \mathrm{C}$ for $5 \mathrm{~min}$ in sample buffer [0.5 M Tris- $\mathrm{HCl}(\mathrm{pH} 6.8), 4 \%$ SDS, $20 \%$ glycerol, $0.1 \%$ bromophenol blue, $10 \% \beta$-mercaptoethanol]. Equal amount of the total proteins were subjected to 6-15\% SDS-PAGE and transferred to PVDF. The membranes were blocked with 5\% non-fat dry milk in Tris-buffered saline with Tween-20 buffer (TBS-T) $(20 \mathrm{mM}$ Tris, $100 \mathrm{mM} \mathrm{NaCl}$, $\mathrm{pH} 7.5$ and $0.1 \%$ Tween-20) for $1 \mathrm{~h}$ at room temperature. Then, the membranes were incubated overnight at $4{ }^{\circ} \mathrm{C}$ with the primary antibodies. The membranes were washed 4 times for $10 \mathrm{~min}$ with TBS-T buffer and then incubated for $1 \mathrm{~h}$ with horseradish peroxidase-conjugated anti-rabbit or anti-mouse immunoglobin (Santa Cruz Biotechnology Inc., Santa Cruz, CA, USA). The membranes were washed again for $10 \mathrm{~min}$ with TBS-T buffer. Antigen-antibody complexes were detected by the enhanced chemiluminescence (ECL) detection system (GE Healthcare Biosciences, Pittsburgh, PA, USA).

Statistical analysis. Measurements of cell viability of RAW 264.7 cells and multiplicity of colonic lesions were analyzed using Student's t-test. The statistical analysis of mRNA expression was performed by the Kruskal-Wallis test. P-value of $<0.05$ was considered to be statistically significant.

\section{Results}

Betaine inhibits AOM/DSS-induced colitis-associated tumorigenesis. The AOM/DSS model is a widely used inflammation-associated colon cancer model in rodents (18-22). In the present study, antitumor effect of dietary administration of betaine was evaluated in AOM/DSS-induced tumorigenesis model. The study protocol is summarized in Fig. 1A. During the experiments, feeding the mice with the different doses of betaine did not produce any observable clinical toxicity or significant changes in body weight compared to control (Fig. 1B). There were no relative changes in colon length between AOM/DSS-induced tumorigenesis groups and betaine-containing diets groups (Fig. 1C and D). Although slight changes were found in colon length, other positive effects such as tumor incidence and inflammatory cytokines were observed on betaine-treated groups with AOM/ DSS-induced tumorigenesis.

Macroscopically, the AOM/DSS model resulted in $100 \%$ incidence of colonic tumors, which were most frequently observed in the middle and distal colon (Fig. 1C, group 2, arrow). The colonic tumors developed in the mice of groups 2 through 5 with different incidence rate and multiplicity (Fig. 2A). AOM/DSS group (group 2) had mainly adenocarcinoma (ADC) with a multiplicity of $9.67 \pm 5.03$. The incidence of ADC in betaine fed (groups 3-5) was less than that of group 2 and the multiplicity of ADC in groups 3, 4 and 5 is $7.03 \pm 2.69$, $5.25 \pm 2.24$ and $3.48 \pm 1.41$, respectively. The multiplicity of colonic ADC in groups 3, 4 and 5 was significantly smaller than group 2 (p<0.01) (Fig. 2A). In H\&E staining, group 2 (AOM/ DSS group) animals showed increased high grade dysplasia, colonic adenoma and tissue inflammation, but administration of betaine resulted in reduction of all of these phenomena in groups 3-5 compared to group 2 dose-dependently (Fig. 2B).

Betaine suppresses inflammatory mediators. Expression of inflammation-associated genes was further confirmed in colonic mucosa by using real-time PCR. AOM/DSS upregulated the mRNA expression of pro-inflammatory genes such as TNF- $\alpha$,IL-6, iNOS and COX-2, compared with group 1 (control group). In contrast, when betaine was given to mice, mRNA expression of TNF- $\alpha$, IL- 6 , iNOS and COX-2 was decreased when compared with the group 2 (AOM/DSS group) (Fig. 3). The expression level of TNF- $\alpha$ and iNOS was decreased 2.5and 5.25 -fold, respectively, compared to group 2 . These results suggest that administration of betaine could reduce inflammation, which may contribute, at least in part, the anticancer effect in AOM/DSS-induced colon tumorigenesis.

Betaine inhibits $R S$ generation and GSSG concentration. Previous studies have shown that betaine suppressed aging-related inflammatory status through inhibition of RS generation and modulation of glutathione (13-15). Thus, we determined the effect of betaine on AOM/DSS-induced RS generation in colonic mucosa using DCF-DA, which is oxidized by RS to fluorescence DCF. As shown in Fig. 4A, the RS formation increased due to AOM/DSS group (group 2). In contrast, betaine-treated groups (groups 3-5) showed decreased RS generation in colonic mucosa. Glutathione is a key intracellular thiol composed of glutamic acid, cysteine, and glycine. Glutathione protects cells from free radical damage by acting as an antioxidant. Within cells, glutathione exists in reduced (GSH) and oxidized (GSSG) states. In healthy cell and tissue, more than $90 \%$ of the total glutathione pool is in the reduced form (GSH) while less than $10 \%$ exists in the disulfide form (GSSG). Thus, GSH depletion can cause redox imbalance through increased oxidative stress (23). To confirm whether betaine can modulate the glutathione levels, we measured GSSG concentration in the homogenate from colonic mucosa. AOM/DSS (group 2) was increased in GSSG concentration compared to group 1 (control group). However, betaine-treated groups (groups 3-5) showed decreased GSSG concentration compared to group 2 (Fig. 4B). Taken together, in part, betaine can overcome oxidative stress including AOM/DSS-induced tumorigenesis through modulation of RS and glutathione levels.

Betaine inhibits LPS-induced pro-inflammatory gene expression in RAW 264.7 cells. After evaluation of anti-inflammatory activity of betaine in the AOM/DSS-induced mouse colon cancer model, we examined its effect on expression of proinflammatory genes in macrophages upon stimulation with lipopolysaccharide (LPS), which is one of the most potent pro-inflammatory agonists for monocytes and macrophages. Betaine pretreatment down-regulated LPS-induced expression of p50, p65, iNOS and COX-2 protein levels in murine macrophage RAW 264.7 cells (Fig. 5). No cytotoxicity was observed under the experimental condition (data not shown). These data suggested that betaine may regulate the macrophage functions in AOM/DSS-induced mouse colon cancer, thereby decreasing pro-inflammatory cytokine productions. 

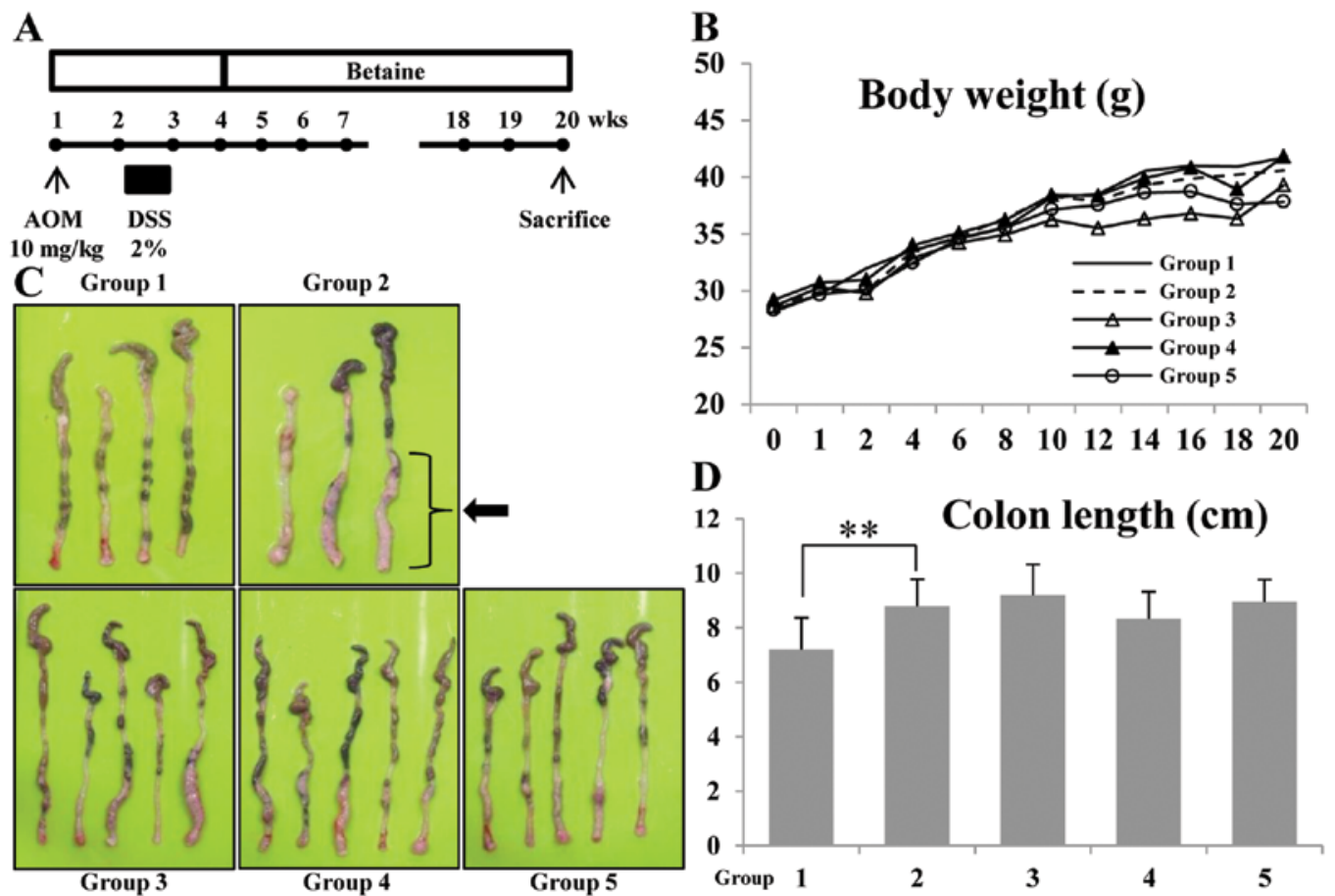

Figure 1. Administration of betaine suppressed AOM/DSS-induced colon carcinogenesis. (A) Experimental protocols. (B) Body weight changes in mice. (C) Representative macroscopic views of the colon of mice. (D) Comparison of the colon length at 16 week in control colon (group 1, control), AOM/DSS-treated mice (group 2, the arrow indicates colonic tumors), and AOM/DSS with different doses (1,5 and $10 \mathrm{mg} / \mathrm{kg}$ in diet) of betaine (groups 3-5). ${ }^{* *} \mathrm{p}<0.01$ compared with the AOM/DSS treatment.
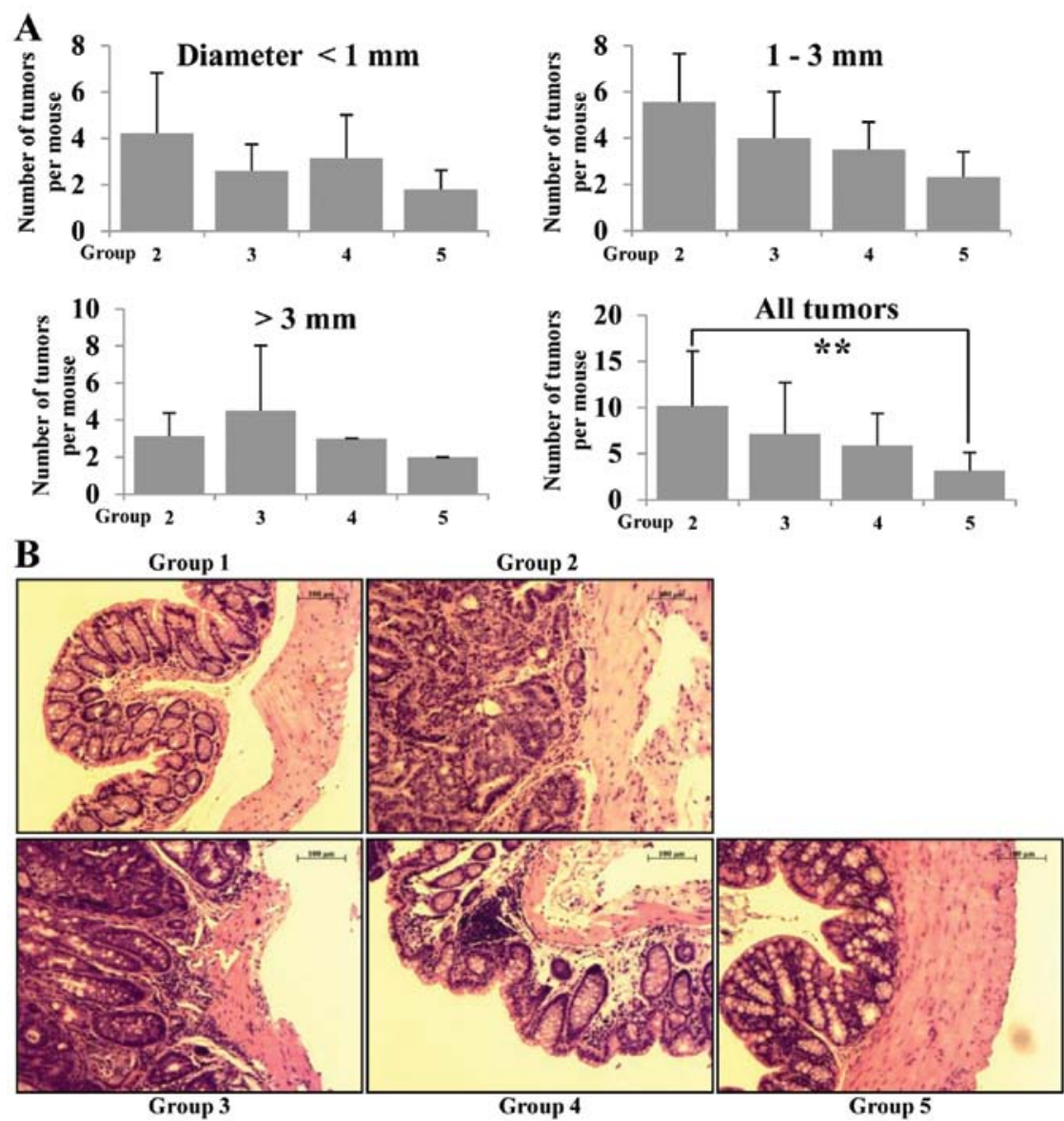

Figure 2. Betaine-containing diets decreased AOM/DSS-induced tumor incidence with inflammation. (A) Incidence and size of colitis-associated colon tumors. * $\mathrm{p}<0.01$ compared with the AOM/DSS treatment (group 2). (B) Representative histopathological images of control colon (group 1), colonic proliferation lesions developed in mice that received AOM/DSS (group 2) and AOM/DSS with different doses of betaine (groups 3-5). H\&E stain. Bar, $100 \mu \mathrm{m}$. 

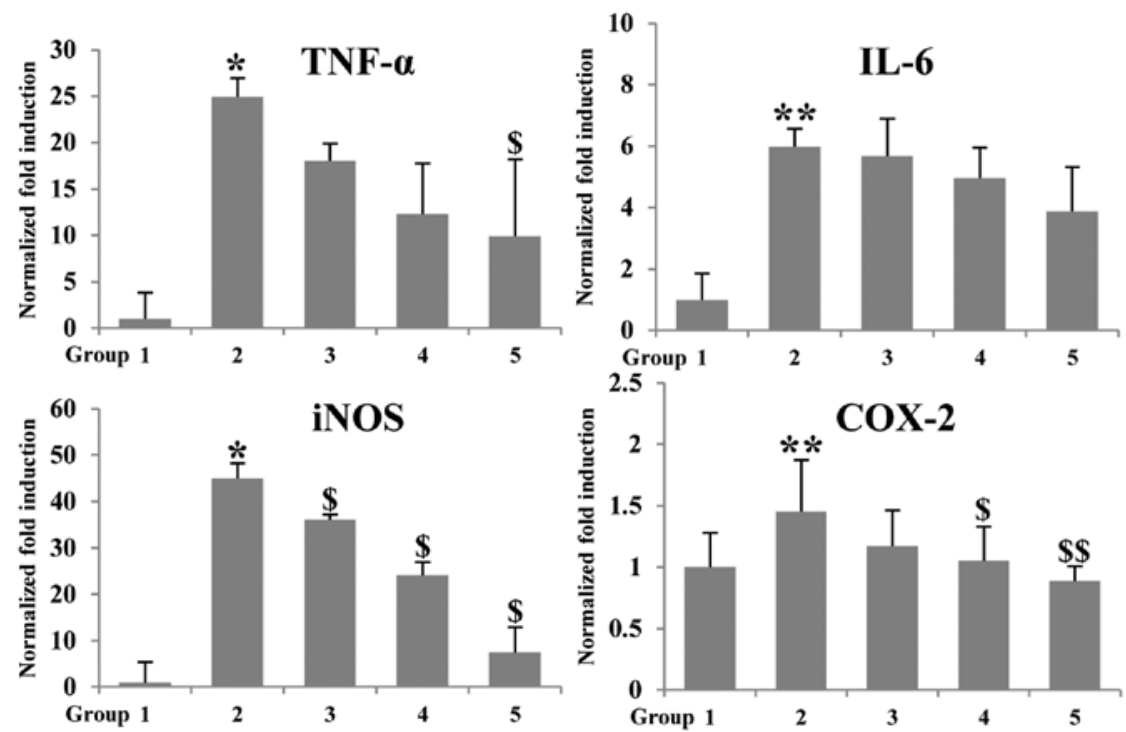

Figure 3. Treatment with betaine inhibited AOM/DSS-induced inflammatory-related cytokines in colonic mucosa. Total RNA was extracted from colonic mucosa from control (group 1), AOM/DSS (group 2) and AOM/DSS followed by various doses of betaine fed mice (groups 3-5). Real-time PCR analyses were carried out by using specific gene primers. ${ }^{*} \mathrm{p}<0.05,{ }^{* *} \mathrm{p}<0.01$ versus the control group. ${ }^{\$} \mathrm{p}<0.05,{ }^{\$ \$} \mathrm{p}<0.01$ versus the AOM/DSS group.
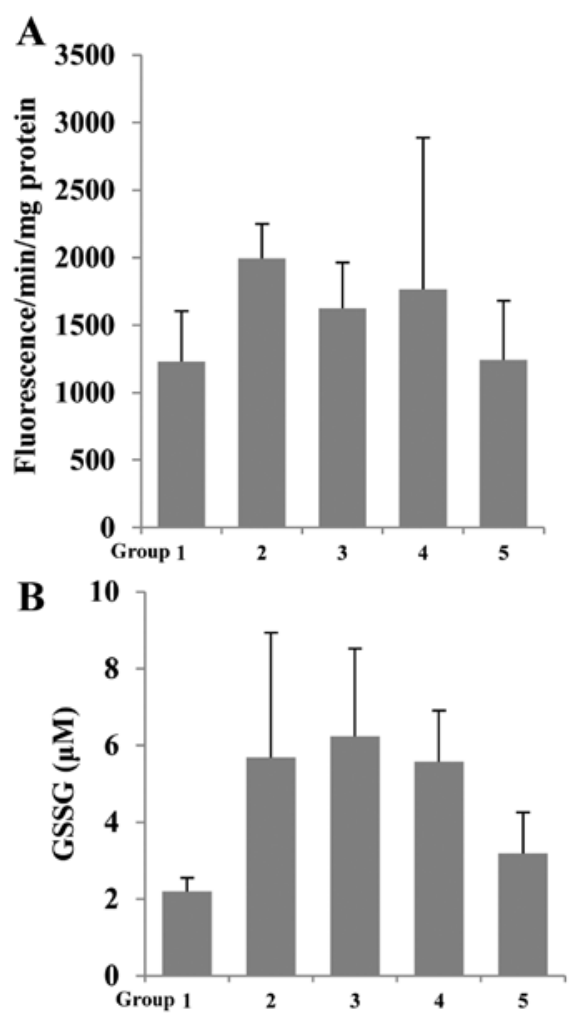

Figure 4. Treatment with betaine inhibits AOM/DSS-induced RS generation and GSSG levels. (A) The DCF-DA method was used to determine the effect of betaine on AOM/DSS-induced RS generation in colonic mucosa homogenates. Each value is the mean \pm SD of five mice $(n=5)$. (B) The GSSG levels in colonic mucosa homogenates were determined as described in Materials and methods. Each value is the mean \pm SD of five mice $(n=5)$.

\section{Discussion}

Inflammation is an important tumor promoter, and several cytokines including TNF- $\alpha$, IL-6, induced by inflammation

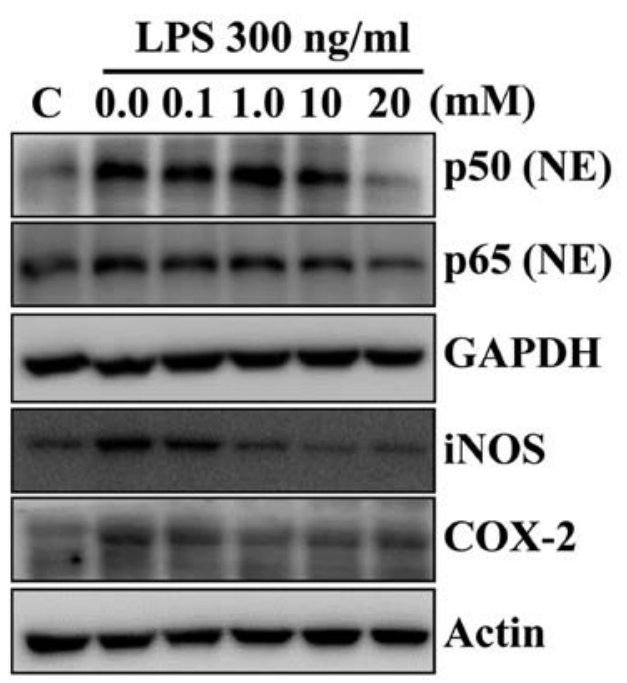

Figure 5. Treatment with betaine inhibited LPS-induced proinflammatory gene expression in murine macrophage RAW 264.7 cells. The cells were pretreated with betaine for $2 \mathrm{~h}$, and then treated LPS for $24 \mathrm{~h}$. Western

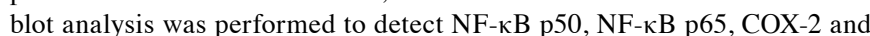
iNOS in RAW 264.7 cells. Representative results from three independent experiments are shown. GAPDH and actin were used as a loading control. C, control.

can promote tumor growth $(18,24)$. Many proto-oncogenes and carcinogens cause activation of $\mathrm{NF}-\kappa \mathrm{B}$, whereas chemicals with known chemopreventive properties can suppress NF- $\kappa \mathrm{B}$ activation (25). Previous studies showed treatment with betaine inhibited NF- $\mathrm{B}$ activation through modulation of ROS and thiol homeostasis during aging process in vitro and in vivo (13-15). In this study, we demonstrated that three doses of betaine administration $(1,5$, and $10 \mathrm{mg} / \mathrm{kg}$ in diet) inhibited colitis-associated colon tumorigenesis in ICR male mice.

Choline and its oxidation product betaine are nutrients involved in one-carbon metabolism (26). Betaine is a nutrient 
abundant in animal foods, especially seafoods, and plant foods including wheat bran and spinach (27). Betaine can donate a methyl group to homocysteine to methionine. It serves as an osmolyte that regulates cell volume and protect cells and protein from environmental stresses including ionic stress (28). Methylation of homocyteine by betaine is confined to the liver and the kidney, but the pathway involving folate exists in all body cells (29). Betaine has been reported as a nutrient preventing inflammatory processes by blocking the expression of pro-inflammatory genes as a consequence of suppressing the $\mathrm{NF}-\kappa \mathrm{B}$ activation in aging process (13). It also suppresses the production of RS and modulation of GSH levels (14). So far, there are limited studies on intake of choline and betaine and cancer risk in humans, because food composition data were not available until recently (30). Several epidemiologic studies have examined the association between dietary intake of choline and betaine and cancer risk. Especially, higher betaine intake, may be protective against lung cancer through mitigating the adverse effect of smoking (31). In the current study, we found that administration of betaine inhibited tumor incidence with inflammation in AOM/DSS-induced colon tumorigenesis in ICR male mice (Fig. 2A). During the experiments, betaine-containing diets did not show any cytotoxicity regarding body weight or food intake. Unfortunately, we did not observe difference of colon length compared to AOM/DSS group (group 2) and betainetreated groups (groups 3-5). However, other factors including gene expression of pro-inflammatory mediators, oxidative stress status (e.g., RS generation and GSSG concentration), and H\&E staining results, were affected by betaine treatment. The qPCR data showed that AOM/DSS-induced inflammatory cytokines including TNF- $\alpha$, IL-6, COX-2 and iNOS were inhibited by betaine treatment in colonic mucosa (Fig. 3). Furthermore, H\&E staining data showed administration of betaine (groups 3-5) decreased AOM/DSS-induced inflammatory-related damage in colonic mucosa compared to group 2 (Fig. 2B).

Redox homeostasis plays a critical role in the protection of cells from both internal and external oxidative and other forms of stress, and it maintains the regulatory role of redox-sensitive transcription factors including NF- $\kappa \mathrm{B}(32,33)$. A previous study showed ROS to play an important role in cancer development, both in the initiation and promotion stages of carcinogenesis (34). In the multi-step process of colon carcinogenesis, ROS were also found to enhance colon carcinogenesis at all stages (35). It has been reported that carcinogenic metals, such as $\mathrm{As}(\mathrm{III})$ and/or $\mathrm{Cr}(\mathrm{VI})$, in drinking water promoted tumorigenesis in murine AOM/DSS colitis-associated colorectal cancer model through modulation of the redox status. Importantly, ROS-mediated $\beta$-catenin activation by carcinogenic metals, As(III) and/or Cr(VI), may play an important role in this promotion effect (36). Betaine has been reported to prevent lysophosphatidylcholine-triggered RS generation and NF- $\kappa \mathrm{B}$ activation in endothelial cells (15). In addition, a previous study reported that dietary betaine supplementation was capable of restoring the redox balance by maintaining thiol homeostasis, thereby suppressing pro-inflammatory $\mathrm{NF}-\kappa \mathrm{B}$ activation during aging (14). Betaine-treated groups showed inhibition of AOM/DSS-induced RS generation and GSSG levels in colonic mucosa (Fig. 4). Therefore, in part, betaine can reduce oxidative stress by modulation of GSSG levels in various types of stress.
LPS is an endotoxin released by gram-negative bacteria that can be transferred to cluster of differentiation 14 by LPS-binding protein and recognized by Toll-like receptor 4 on the cellular surface of macrophages (37). LPS triggers the translocation of NF- $\kappa \mathrm{B}$ lead to the expression of NF- $\kappa \mathrm{B}$-regulated genes including TNF- $\alpha$, IL-6, COX-2 and iNOS in murine macrophage RAW 264.7 cells (38). Our results demonstrated that betaine treatment inhibited the LPS-induced TNF- $\alpha$, IL-6, COX-2 and iNOS in RAW 264.7 cells (Fig. 5).

In conclusion, administration with betaine effectively suppressed AOM/DSS-induced mouse colon tumor incidence with inflammation by suppressing the expression of cytokines, such as TNF- $\alpha$, IL-6, COX-2 and iNOS. In addition, treatment with betaine decreased RS generation and modulation of total glutathione concentration. Collectively, betaine is a candidate cancer chemopreventive agent against cancer development in inflammation-associated colon tumorigenesis.

\section{Acknowledgements}

This study was supported by the National Research Foundation of Korea (NRF) grant funded by the Korea government (MSIP) (no. 2009-0083538) and the R\&D Program of MKE/KEIT (10040391, Development of Functional Food Materials and Device for Prevention of Aging-associated Muscle Function Decrease). We thank Aging Tissue Bank for providing research information.

\section{References}

1. Itzkowitz SH and Yio X: Inflammation and cancer IV. Colorectal cancer in inflammatory bowel disease: the role of inflammation. Am J Physiol Gastrointest Liver Physiol 287: G7-G17, 2004.

2. Loftus EV Jr: Clinical epidemiology of inflammatory bowel disease: Incidence, prevalence, and environmental influences. Gastroenterology 126: 1504-1517, 2004.

3. Hou JK, El-Serag H and Thirumurthi S: Distribution and manifestations of inflammatory bowel disease in Asians, Hispanics, and African Americans: a systematic review. Am J Gastroenterol 104: 2100-2109, 2009.

4. Yang SK, Yun S, Kim JH, et al: Epidemiology of inflammatory bowel disease in the Songpa-Kangdong district, Seoul, Korea, 1986-2005: a KASID study. Inflamm Bowel Dis 14: 542-549, 2008.

5. Pedersen J, Coskun M, Soendergaard C, Salem M and Nielsen OH: Inflammatory pathways of importance for management of inflammatory bowel disease. World J Gastroenterol 20: 64-77, 2014.

6. Nielsen $\mathrm{OH}$, Seidelin JB, Munck LK and Rogler G: Use of biological molecules in the treatment of inflammatory bowel disease. J Intern Med 270: 15-28, 2011.

7. Targownik LE and Bernstein CN: Infectious and malignant complications of TNF inhibitor therapy in IBD. Am J Gastroenterol 108: 1835-1842, quiz 1843, 2013.

8. Craig SA: Betaine in human nutrition. Am J Clin Nutr 80: 539-549, 2004.

9. Steenge GR, Verhoef P and Katan MB: Betaine supplementation lowers plasma homocysteine in healthy men and women. J Nutr 133: 1291-1295, 2003.

10. Kim SK and Kim YC: Attenuation of bacterial lipopolysaccharide-induced hepatotoxicity by betaine or taurine in rats. Food Chem Toxicol 40: 545-549, 2002.

11. Zeisel SH and Blusztajn JK: Choline and human nutrition. Annu Rev Nutr 14: 269-296, 1994.

12. Detopoulou P, Panagiotakos DB, Antonopoulou S, Pitsavos C and Stefanadis C: Dietary choline and betaine intakes in relation to concentrations of inflammatory markers in healthy adults: the ATTICA study. Am J Clin Nutr 87: 424-430, 2008. 
13. Go EK, Jung KJ, Kim JY, Yu BP and Chung HY: Betaine suppresses proinflammatory signaling during aging: the involvement of nuclear factor-kappaB via nuclear factorinducing kinase/IkappaB kinase and mitogen-activated protein kinases. J Gerontol A Biol Sci Med Sci 60: 1252-1264, 2005.

14. Go EK, Jung KJ, Kim JM, et al: Betaine modulates age-related NF-kappaB by thiol-enhancing action. Biol Pharm Bull 30: 2244-2249, 2007.

15. Lee EK, Jang EJ, Jung KJ, Kim DH, Yu BP and Chung HY: Betaine attenuates lysophosphatidylcholine-mediated adhesion molecules in aged rat aorta: modulation of the nuclear factorkappaB pathway. Exp Gerontol 48: 517-524, 2013.

16. Kim DH, Hossain MA, Kang YJ, et al: Baicalein, an active component of Scutellaria baicalensis Georgi, induces apoptosis in human colon cancer cells and prevents AOM/DSS-induced colon cancer in mice. Int J Oncol 43: 1652-1658, 2013.

17. LeBel CP, Ischiropoulos $\mathrm{H}$ and Bondy SC: Evaluation of the probe 2',7'-dichlorofluorescin as an indicator of reactive oxygen species formation and oxidative stress. Chem Res Toxicol 5: 227-231, 1992.

18. Tanaka T, Kohno H, Suzuki R, Yamada Y, Sugie S and Mori H: A novel inflammation-related mouse colon carcinogenesis model induced by azoxymethane and dextran sodium sulfate. Cancer Sci 94: 965-973, 2003.

19. Kim M, Miyamoto S, Sugie S, et al: A tobacco-specific carcinogen, NNK, enhances AOM/DSS-induced colon carcinogenesis in male A/J mice. In Vivo 22: 557-563, 2008.

20. Tanaka T, Yasui Y, Tanaka M, Oyama T and Rahman KM: Melatonin suppresses AOM/DSS-induced large bowel oncogenesis in rats. Chem Biol Interact 177: 128-136, 2009.

21. Miyoshi N, Nagasawa T, Mabuchi R, et al: Chemoprevention of azoxymethane/dextran sodium sulfate-induced mouse colon carcinogenesis by freeze-dried yam sanyaku and its constituent diosgenin. Cancer Prev Res (Phila) 4: 924-934, 2011.

22. Yasui Y, Hosokawa M, Mikami N, Miyashita K and Tanaka T: Dietary astaxanthin inhibits colitis and colitis-associated colon carcinogenesis in mice via modulation of the inflammatory cytokines. Chem Biol Interact 193: 79-87, 2011.

23. Bizzozero OA, Ziegler JL, De Jesus G and Bolognani F: Acute depletion of reduced glutathione causes extensive carbonylation of rat brain proteins. J Neurosci Res 83: 656-667, 2006.

24. Tanaka T: Colorectal carcinogenesis: Review of human and experimental animal studies. J Carcinog 8: 5, 2009.

25. Bharti AC and Aggarwal BB: Chemopreventive agents induce suppression of nuclear factor-kappaB leading to chemosensitization. Ann NY Acad Sci 973: 392-395, 2002
26. Lee JE, Giovannucci E, Fuchs CS, Willett WC, Zeisel SH and Cho E: Choline and betaine intake and the risk of colorectal cancer in men. Cancer Epidemiol Biomarkers Prev 19: 884-887, 2010.

27. Konstantinova SV, Tell GS, Vollset SE, Nygard O, Bleie O and Ueland PM: Divergent associations of plasma choline and betaine with components of metabolic syndrome in middle age and elderly men and women. J Nutr 138: 914-920, 2008.

28. Teixido N, Canamas TP, Usall J, Torres R, Magan N and Vinas I: Accumulation of the compatible solutes, glycinebetaine and ectoine, in osmotic stress adaptation and heat shock cross-protection in the biocontrol agent Pantoea agglomerans CPA-2. Lett Appl Microbiol 41: 248-252, 2005.

29. Olthof MR and Verhoef P: Effects of betaine intake on plasma homocysteine concentrations and consequences for health. Curr Drug Metab 6: 15-22, 2005.

30. Ueland PM: Choline and betaine in health and disease. J Inherit Metab Dis 34: 3-15, 2011

31. Ying J, Rahbar MH, Hallman DM, et al: Associations between dietary intake of choline and betaine and lung cancer risk. PLoS One 8: e54561, 2013.

32. Liu H, Colavitti R, Rovira II and Finkel T: Redox-dependent transcriptional regulation. Circ Res 97: 967-974, 2005.

33. Palozza P, Serini S, Torsello A, et al: Beta-carotene regulates NF-kappaB DNA-binding activity by a redox mechanism in human leukemia and colon adenocarcinoma cells. J Nutr 133: 381-388, 2003.

34. Pelicano H, Carney D and Huang P: ROS stress in cancer cells and therapeutic implications. Drug Resist Updat 7: 97-110, 2004.

35. Erdelyi I, Levenkova N, Lin EY, et al: Western-style diets induce oxidative stress and dysregulate immune responses in the colon in a mouse model of sporadic colon cancer. J Nutr 139: 2072-2078, 2009.

36. Wang X, Mandal AK, Saito $\mathrm{H}$, et al: Arsenic and chromium in drinking water promote tumorigenesis in a mouse colitis-associated colorectal cancer model and the potential mechanism is ROS-mediated Wnt/beta-catenin signaling pathway. Toxicol Appl Pharmacol 262: 11-21, 2012.

37. Takeda $\mathrm{K}$ and Akira S: Toll-like receptors in innate immunity. Int Immunol 17: 1-14, 2005

38. Ci X, Ren R, Xu K, et al: Schisantherin A exhibits anti-inflammatory properties by down-regulating NF-kappaB and MAPK signaling pathways in lipopolysaccharide-treated RAW 264.7 cells. Inflammation 33: 126-136, 2010 